\title{
A Novel Combination Method for Image Restoration Based on Bootstrap Filter and DWT
}

\author{
Bassel Marhaba ${ }^{1, *}$, Mourad Zribi $^{1}$ \\ ${ }^{1}$ Université du Littoral Côte d’Opale, 62228 Calais Cedex France.
}

\begin{abstract}
In our paper, we propose a novel combination of two methods based on the bootstrap filter. We will use the fusion technique to combine the restored images from the bootstrap multivariate kernel density filter and the bootstrap kernel-diffeomorphism filter. Experimental results have shown that our method has proved the image restoration results.
\end{abstract}

\section{Introduction}

Image restoration is useful in many fields such as satellites, medical devices, consumer camera devices and robotics devices by eliminating the noise effect in the captured images and videos. Speckle noise is considered. In our paper, we propose to use a fusion combination, based on the discrete wavelet transform (DWT) [1], between the bootstrap multivariate kernel density filter (BMKDF) [2] and the bootstrap kernel diffeomorphism filter (BKDF) [3] in order to get a new image with a better restoration results.

\section{The image model and the Bayesian bootstrap filter}

Let the original image $s(n, m)$ with $m$ rows and $n$ columns, be represented by the image model:

$$
s(m, n)=\sum_{(k, l) \in \psi} c_{k, l} s(m-k, n-l)+w(m, n)
$$

where, $c_{k, l}$ are the coefficients of the image, $\psi$ is the image model support, and $w(m, n)$ is a zero-mean noise with covariance $\sigma_{w}^{2}(m, n)$, and let the state vector $x(m, n)$ at a location $(m, n)$ be:

$$
x(m, n)=\left[\begin{array}{l}
s(m, n), s(m-1, n), \ldots, s(1, n) ; \\
s(N, n-1), \ldots, s(1, n-1) ; \ldots, \\
s(N, n-M), \ldots, s(m-M, n-M)
\end{array}\right]^{T}
$$

Now let the degraded image $y(m, n)$ be:

$$
y(m, n)=s(m, n) v(m, n)
$$

\footnotetext{
*bassel.marhaba@etu.univ-littoral.fr
} 
where $v(m, n)$ is the speckle noise with variance of $\sigma_{v}^{2}$ and a unit mean. The aim of BBF [2] is to estimate the posterior pdf $p\left(x(m, n) \mid Y_{m, n}\right)$ from a set of random samples $\left\{x_{i}(m-1, n): i=1, \ldots, N\right\}$ which are drawn using the pdf $p(x(m-1, n) \mid Y(m-1, n))$, where $N$ is the number of bootstrap samples. Here each sample will go through the system transition function $x(m, n)=b(x(m-1, n), w(m-1, n))$ to obtain a new sample $x_{i}^{*}(m, n)$. A normalized weight $q_{i}$ for each sample will be obtained by:

$$
q_{i}=\frac{p\left(y(m, n) \mid x_{i}^{*}(m, n)\right)}{\sum_{j=1}^{N} p\left(y(m, n) \mid x_{j}^{*}(m, n)\right)}
$$

Now we will resample $N$ times to get the samples $\left\{x_{i}(m, n): i=1, \ldots, N\right\}$, so that for any $j, \operatorname{Prob}\left\{x_{j}(m, n)=x_{i}^{*}(m, n)\right\}=q_{i}$. We assume that these samples are distributed as the required PDF $p\left(x(m, n) \mid Y_{m, n}\right)$ given by:

$$
p\left(x(m, n) \mid Y_{m, n}\right)=\sum_{i=1}^{N_{b}} q_{i} \delta\left(x(m, n)-x_{i}(m, n)\right)
$$

\section{The Bootstrap Multivariate Kernel Density Filter (BMKDF) and the Bootstrap Kernel-Diffeomorphism Filter (BKDF)}

The function that defines the kernel density estimation is [2]:

$$
\hat{f}_{H}(x)=\frac{1}{n} \sum_{i=1}^{n} K_{H}\left(x-x_{i}\right)
$$

where $H$ assumed to be the bandwidth matrix of dimensions $d \times d$ and is assumed to be symmetric and positive. The standard multivariate kernel density function $K$ is defined as:

$$
K_{H}(x)=(2 \pi)^{-d / 2}|H|^{-1 / 2} e^{-\frac{1}{2} x^{T} H^{-1} x}
$$

where $H$ acts as the covariance matrix. By using a multivariate kernel density estimation, The BBF will be BMKDF and Eq. (5) will be:

$$
p\left(x(m, n) \mid Y_{m, n}\right)=\sum_{i=1}^{N_{b}} q_{i} K_{H}\left(x(m, n)-x_{i}(m, n)\right)
$$

where $K_{H}$ is defined by (7).

The estimated density $\hat{d}_{X, M}(x)$ for a multivariate data $x \in U$ will be given by [3]:

$$
\hat{d}_{X, M}(x)=\frac{\left|\operatorname{det}\left\{\phi^{\prime}(x)\right\}\right|}{M\left(h_{M}\right)^{p}} \sum_{m=1}^{M} K\left(\frac{\phi(x)-\phi\left(X_{m}\right)}{h_{M}}\right)
$$

In BKDF, the posterior $p\left(x(m, n) \mid Y_{m, n}\right)$ in eq (5) will be given by:

$$
p\left(x(m, n) \mid Y_{m, n}\right)=\frac{\left|\operatorname{det}\left\{\phi^{\prime}(x(m, n))\right\}\right|}{N_{b}\left(h_{N_{b}}\right)^{p}} \sum_{n=1}^{N_{b}} q_{i} K\left(\frac{\phi(x(m, n))-\phi\left(x_{i}(m, n)\right)}{h_{N_{b}}}\right)
$$




\section{The Proposed Method}

Here, we propose to combine the same image restored with the BMKDF and the BKDF using fusion method based on the DWT [1]. This procedure will be processed as in Fig. 1:

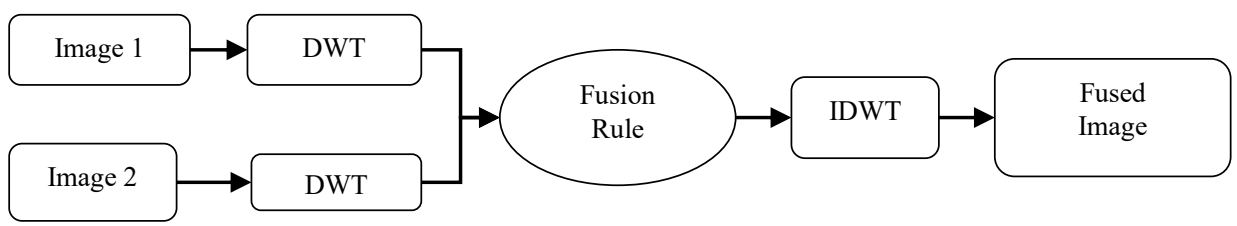

Fig. 1: Fusion method based on DWT

The effective work in the wavelet-based image fusion is to combine the coefficients such a way to have the best quality of the fused image. There are many ways to achieve this goal; the simplest way is to calculate the average of the coefficients to be integrated.

\section{The Experimental Results}

To illustrate the superior of the proposed method we have chosen the city of Amsterdam SAR image as an example. We simulate a noisy image with speckle noise with unity mean and variance of 0.09. In Fig. 2 we illustrate the visual results, and in Table 1 we illustrate the metric results.

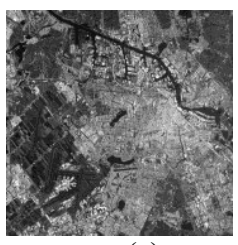

(a)

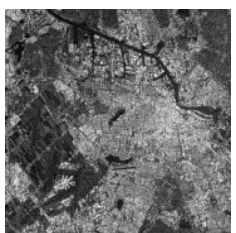

(b)

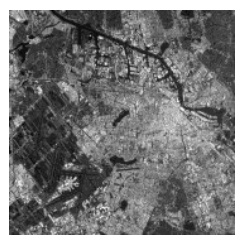

(c)

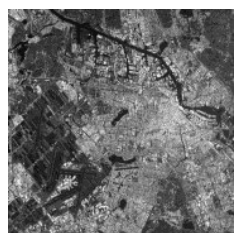

(d)

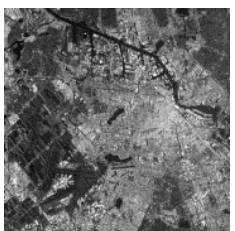

(e)

Fig. 2: (a) Original image (b) Noisy image (c) Denoised by BKDF (d) Denoised by BMKDF (e) Denoised by the proposed method.

Table 1: The PSNR results for the denoised SAR image of Amsterdam for the several methods

\begin{tabular}{|l|l|l|l|}
\hline Filter & BKDF & BMKDF & Proposed \\
\hline PSNR & 25.17 & 26.15 & 27.33 \\
\hline
\end{tabular}

\section{The Conclusion}

In our paper, we proposed the use of fusion technique based on the DWT between two methods, the BKDF and the BMKDF. The proposed method has ameliorated the image restoration results. Our proposed method can be used for all image types and also in video processing for reducing noise in videos.

\section{References}

1. L. Papamarkou, et al, A novel image sharpening technique based on 2D-DWT and image fusion, International Conference on Information Fusion, 1-8, (2014)

2. B. Marhaba and M. Zribi, Regularized Bootstrap Filter for Image Restoration, CGVCVIP, 117-123, (2017)

3. B. Marhaba and M. Zribi, 2018.The bootstrap Kernel-diffeomorphism filter for satellite image restoration, ISCT, 80-84, (2018) 